\title{
Design, simulation and fabrication of a 3D printed pump mimicking the left ventricle motion
}

\section{Emanuele Vignali ${ }^{1,2, *}$, Zaira Manigrasso ${ }^{2, *}$, Emanuele Gasparotti ${ }^{1,2, *}$, Benedetta Biffi ${ }^{3}$, Luigi Landini ${ }^{2}$, Vincenzo Positano ${ }^{1}$, Claudio Capelli ${ }^{3}$ and Simona Celi ${ }^{1}$}

\begin{abstract}
The development of accurate replicas of the circulatory and cardiac system is fundamental for a deeper understanding of cardiovascular diseases and the testing of new devices. Although numerous works concerning mock circulatory loops are present in the current state of the art, still some limitations are present. In particular, a pumping system able to reproduce the left ventricle motion and completely compatible with the magnetic resonance environment to permit the 4D flow monitoring is still missing. The aim of this work was to evaluate the feasibility of an actuator suitable for cardiovascular mock circuits. Particular attention was given to the ability to mimic the left ventricle dynamics including both compression and twisting with the magnetic resonance compatibility. In our study, a left ventricle model to be actuated through vacuum was designed. The realization of the system was evaluated with finite element analysis of different design solutions. After the in-silico evaluation phase, the most suitable design in terms of physiological values reproduction was fabricated through 3D printing for in-vitro validation. A pneumatic experimental setup was developed to evaluate the pump performances in terms of actuation, in particular ventricle radial and longitudinal displacement, twist rotation and ejection fraction. The study demonstrated the feasibility of a custom pneumatic pump for mock circulatory loops able to reproduce the physiological ventricle movement and completely suitable for the magnetic resonance environment.
\end{abstract}

\section{Keywords}

Heart, Mock circulation loop, Pump, 3D printing, Twist rotation, Ejection fraction

\section{Introduction}

The development of new implantable devices in the cardiovascular field is of crucial importance in fighting heart diseases. Currently, for the validation of these technologies, the usage of in-silico and in-vitro techniques has significantly increased. Regarding the in-vitro environment, several mock circulatory loops were proposed in literature (Mechoor et al. (2016); Chaudhury et al. (2016); Lanzarone et al. (2009)). These experimental setups are usually composed by both an active component (the artificial heart pump) and passive elements to reproduce the physiological pressure of the circulatory system. Their main focus is targeted on the simulation of physiological flow in terms of velocity amplitudes, heart cycle times and stroke volumes (Tsai and Savaş (2010); Taylor et al. (2012)). Regarding the commonly adopted active components, electro-mechanical piston or gear pumps are usually implemented to reproduce blood vessel flows, including the ascending aorta. These kind of actuators have demonstrated to be suitable for flow profile reproduction, but the full complexity of the helicoidal nature of the blood stream is neglected. In recent years the adoption of Magnetic Resonance Imaging (MRI) techniques with the 3D phase contrast sequences (3D PC MRI) permitted to visualize and to quantify the flows in-vivo. At the ascending aortic level, the 3D PC- MRI sequences well pointed out the complexity of the blood flow.

This complexity is originated in part by non-planar curvatures of the vessel but mainly by the left ventricle
(LV) movement (Morbiducci et al. (2011)). The LV, in fact, is characterized by twisting and torsion as a consequence of its contraction during the cardiac cycle. The LV is subject to a longitudinal and radial displacement and a twist rotation around its long axis (Sengupta et al. (2008)). This motion sequence contributes to flow efficiency increase at aortic valve level (Beladan et al. (2014)). The LV motion is induced by a system of three layers of fibres: the subepicardium layer, with left-handed orientation; the mid-wall with horizontal orientation; the sub-endocardium with righthanded orientation (Nakatani (2011)). The importance of the fibre architecture for the 3D LV movement was recently reported by Roche and colleagues (Roche et al. (2017)). Given the relevance of the LV morphology, different studies reported designs of pumping systems relying on harvested bovine or swine hearts, however the physiological ventricular twisting could not be achieved with the proposed approach (Leopaldi et al. (2015); Jaworek et al. (2017)). Additionally,

\footnotetext{
${ }^{1}$ BioCardioLab, Ospedale del Cuore, Fondazione Toscana G Monasterio, Massa, Italy

${ }^{2}$ Department of Information Engineering, University of Pisa, Pisa, Italy

${ }^{3}$ UCL Institute of Cardiovascular Science, London, UK

* Contributed equally
}

\section{Corresponding author:}

Simona Celi, BioCardioLab, Ospedale del Cuore, Fondazione Toscana G. Monasterio, Via Aurelia sud, 54100 Massa, Italy

Email: s.celi@ftgm.it 


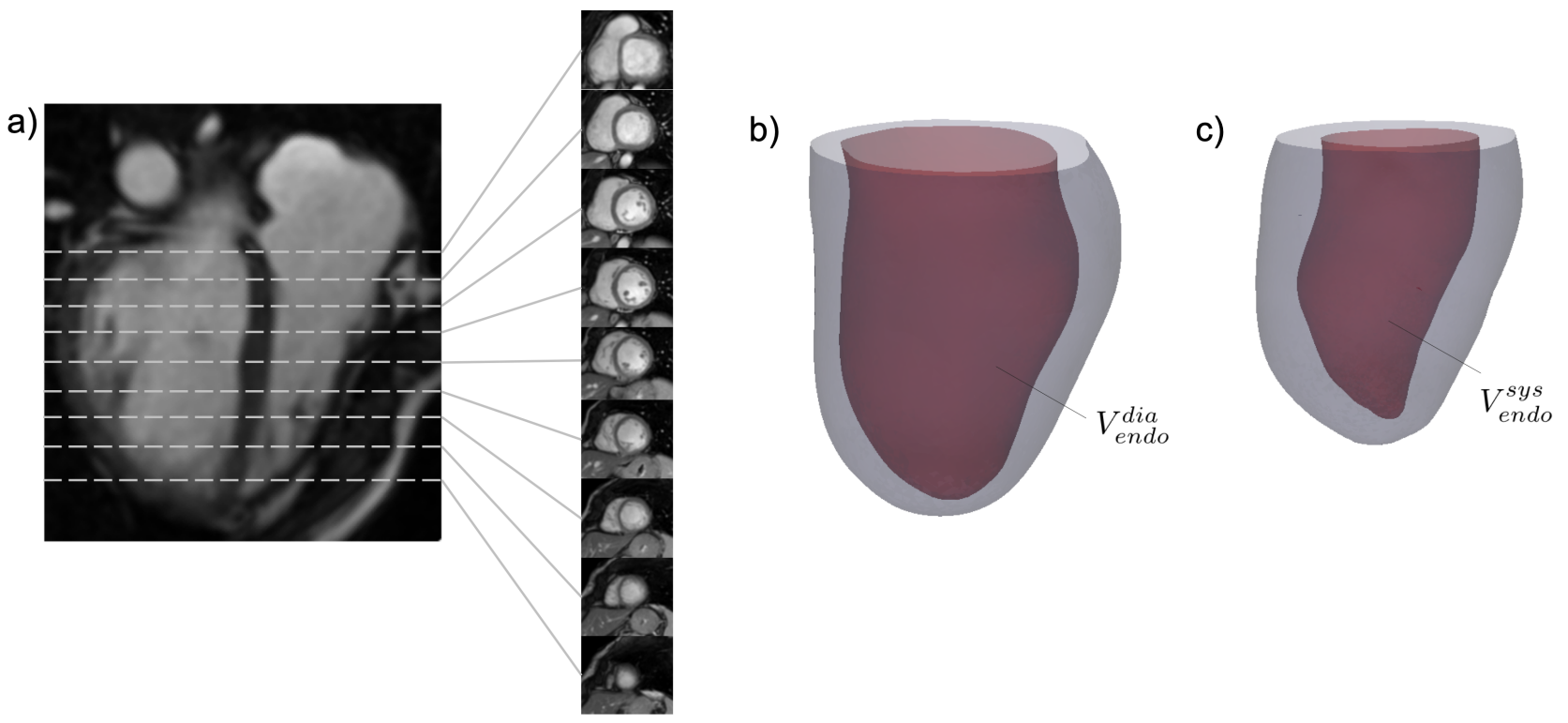

Figure 1. Example of MRI images in long and short axes (a); 3D reconstruction of endocardium and epicardium volume at diastolic (b) and systolic (c) phases.

the adoption of ex-vivo animal tissues brings disadvantages like the necessity to assure the tissue preservation as well as long setting up times, together with potential reproducibility issues and biological samples management.

Advances in medical imaging field brought improvements, not only in terms of functional imaging (i.e. 3D PC-MRI), but also in terms of quality of the morphological information (Celi et al. (2017)). In the context of cardiovascular medicine, a significant added value was provided by the synergy between anatomical imaging and 3D printing technology. The 3D printing techniques entered in the world of cardiovascular medicine in different ways, as they permit the realization of complex structures with customizable material properties (Gasparotti et al. (2018); Marone et al. (2018); Marconi et al. (2018)). Among the main advantages of this technology, of particular importance is the capability to manufacture polymeric materials with a wide range of deformability (i) and to obtain fully MRI compatible structures (ii). These aspects open new paths in the development of novel approaches in the field of mock circulatory loops.

The aim of this work is to demonstrate the feasibility, in terms of fabrication and actuation, of a new pumping system prototype through $3 \mathrm{D}$ printing technique. The following characteristics were taken into account as main specifications: ability to mimic the LV motion of both compression/expansion and rotation, ejection fraction reproduction and compatibility with the MRI environment. To this purpose, both in-silico and in-vitro approaches were adopted to obtain a preliminary design of the system. To fulfill the given specifications, different pump designs were presented. A first phase of in-silico modeling was setup to assess the pump design through Finite Element (FE) analysis of the system actuation. Once the final design was assessed, the LV model was fabricated by 3D printing technique. Subsequently, an experimental setup was defined to demonstrate the system feasibility and performances invitro in terms of actuation through vacuum.

\section{Methods}

The following phases for the pump realization were followed: an anatomy segmentation phase, a geometry definition phase for the different CAD designs, a Finite Element evaluation phase and an experimental testing phase.

\section{Anatomy segmentation}

Steady-state free procession two-chamber, four-chamber and short-axis cine images of an adult healthy subject were acquired (8-mm thickness, 30 phases) using a 1.5-T MR scanner (GE Signa/Excite, Milwaukee, Wisconsin,USA) (Figure 1a). A manual segmentation of the MRI slices was performed to identify endocardium and epicardium contours. The process was carried out by an expert cardiologists following the current guidelines. According to Aquaro et al. (2017) the expected variability in the assessment of EF is less than $5 \%$. The anatomy-based $3 \mathrm{D}$ geometry of the ventricle was reconstructed from endocardial and epicardial contours by using 3D Slicer software. From the reconstructed contours, the epicardium and endocardium surfaces were created at each acquisition phase. A smoothing process was then applied to the surfaces of the ventricle to reduce artifacts induced by the segmentation interpolation (Figure 1b-c). By evaluating the left ventricle volume (i.e. the volume inside the epicardial surface) through the cardiac cycle, the diastolic and systolic phases were identified as the frames with the maximum and minimum volumes, respectively. By comparing the variation of the endocardium volumes from diastole to systole, the ejection fraction $\left(\mathrm{EF}_{\text {seg }}\right)$ was evaluated according to Equation 1:

$$
E F_{\text {seg }}=100\left(\frac{V_{\text {endo }}^{\text {dia }}-V_{\text {endo }}^{\text {sys }}}{V_{\text {endo }}^{\text {dia }}}\right)
$$

where $V_{\text {endo }}^{\text {dia }}$ and $V_{\text {endo }}^{\text {sys }}$ are the endocardium volumes in diastole and systole, respectively. 
a)

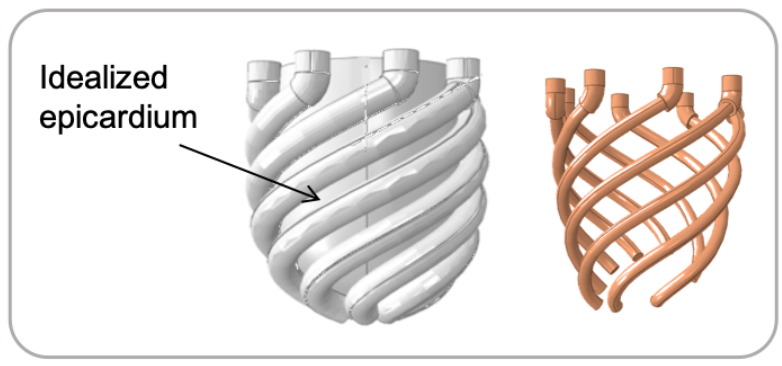

b)

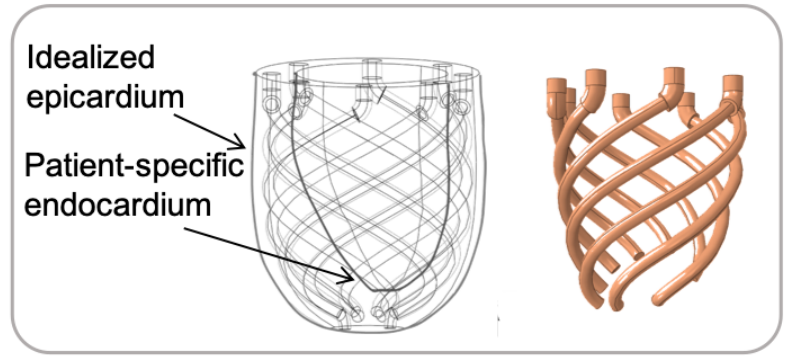

c)

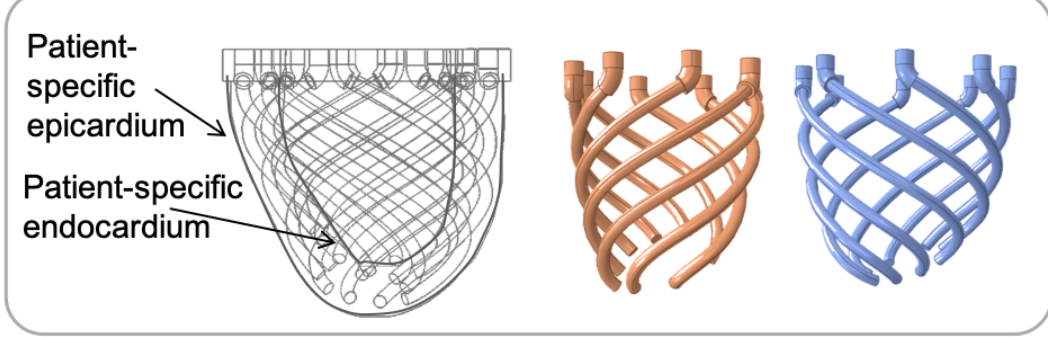

d)

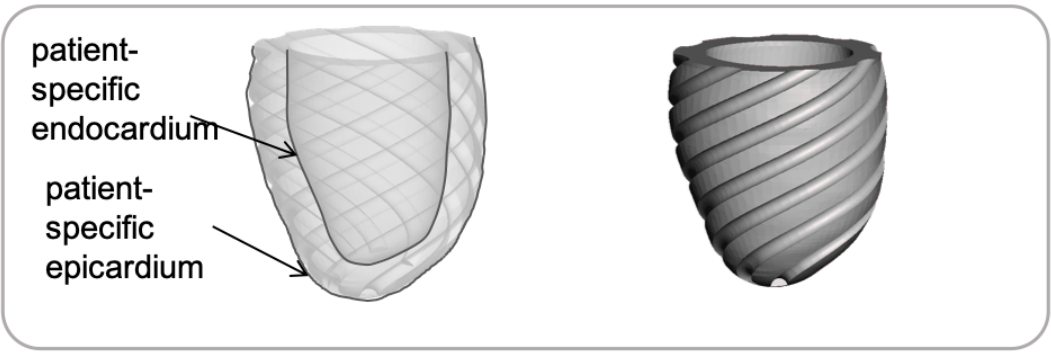

e)

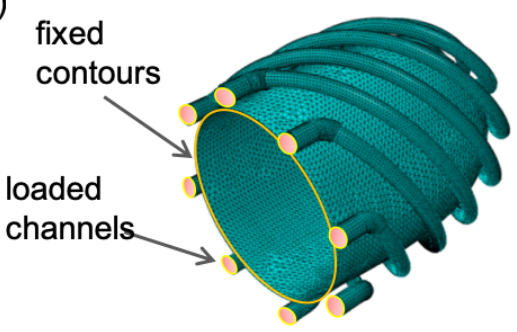

f)

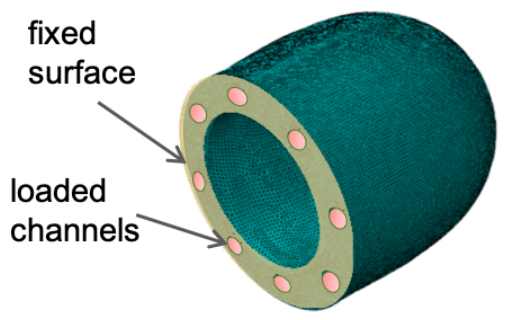

g)

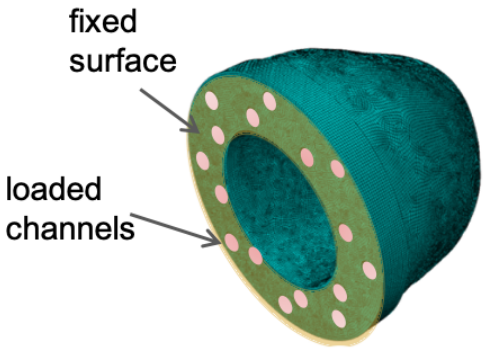

h)

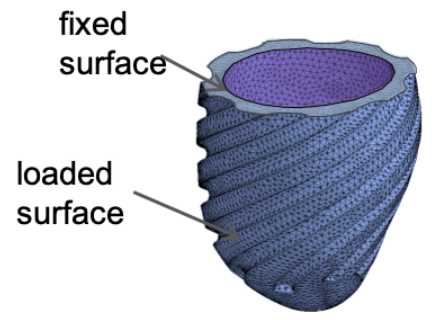

Figure 2. CAD design for the investigated geometries and the corresponding FEM models: $D_{1}$ and $M_{1}(\mathrm{a}-\mathrm{e}), D_{2}$ and $M_{2}$ (b-f), $D_{3}$ and $M_{3}(\mathrm{c}-\mathrm{g}), D_{4}$ and $M_{4}$ (d-h).

\section{Geometrical model}

The segmented surfaces were used as a basis for the definition of the 3D CAD models of the ventricle. The overall geometrical dimensions were set according to those obtained with the segmentation. Four different approaches of pneumatic pump designs were assessed by considering two strategies of ejection: from diastolic volume to systolic volume (compression) and viceversa (expansion). Both the strategies were obtained by the realization of helicoidal structures mimicking the left ventricle muscular fibers, actuated by vacuum. The compression strategy was obtained by applying vacuum inside the helicoidal channels, as the negative pressure causes channel shortening. The expansion strategy was obtained by applying vacuum on the helicoidal grooves, as the negative pressure causes a wall expansion. For all the cases, the main goal was to reproduce both the ejection fraction and the twist angle values at patient specific level. The four designs, developed on the basis of the abovementioned strategies, are presented in Figure 2a-d.

Design $1\left(D_{1}\right)$ : was based on an idealized epicardium and endocardium surface, defined in order to replicate the LV volume in diastole. The actuation was thought to be achieved by negative pressurization of a layer of channels placed on the epicardium surfaces. The channels are designed according to an helical pattern with an orientation angle of $60^{\circ}$. This geometrical and load configuration is set to guarantee the compression and the twist movement of the pump model at the same time. (Figure 2a).

Design $2\left(D_{2}\right)$ : was based on an idealized epicardium and patient-specific endocardium surface in the diastolic phase. Moreover, differently from design $D_{1}$, the layer of channels is placed inside the thickness between the endocardium a and the epicardium. The channels are designed according to the same helical pattern of $D_{1}$. The actuation is given 
by the negative pressurization of the channels to impose a compression an the twisting of the pump model. (Figure 2b).

Design $3\left(D_{3}\right)$ : was based on patient-specific epicardium and endocardium surfaces in the diastolic phase. Differently from the first two designs, two layers of channels are placed inside the thickness between the endocardium and the epicardium. The channel sets are designed according to two symmetrical helicoidal patterns with angles of $\pm 60^{\circ}$. The actuation is given by the negative pressurization of the two sets of channels to obtain both compression and twisting (Figure 2c).

Design $4\left(D_{4}\right)$ : was based on patient-specific epicardium and endocardium surfaces in the systolic phase. Differently to the previous designs, in this case the negative pressurization causes an expansion. No channels are present in the model and the load is applied directly on the epicardium surface. In order to obtain the twist effect during the expansion, a set of specific helical grooves is modeled on the external surface. The set of grooves have a right-handed helical pattern with an orientation angle of $60^{\circ}$ (Figure $2 \mathrm{~d}$ ).

\section{Material testing}

A printable thermopolyurethane was chosen for insilico analysis of the prototype (TangoBlackPlus FLX980, Stratasys Ltd. ${ }^{\complement}$, Tensile Strength: 0.8-1.5 MPa, Elongation at Break: 170 - $220 \%$, Shore Hardness: 26-28 scale A). To assess the material properties (Young modulus and ultimate elongation) of TangoBlackPlus, a set of uniaxial traction tests were performed on five dog-bone specimens according to ASTM D412. A Polyjet 3D printing machine (Objet500 Connex3, Stratasys Ltd.`) was used for specimens fabrication.

\section{Finite element simulations}

FEM structural analysis was performed for each of the design previously described to assess the performances of the different pump models $\left(M_{1}, M_{2}, M_{3}\right.$ and $M_{4}$ ) (Figure 2 e-h). The structural analyses were carried out through the Structural Module of the commercial software ANSYS (ANSYS, Canonsburg, Pennsylvania). The material model was assumed as linear elastic on the basis of the results reported from the mechanical testing of TangoBlackPlus. All the models were constrained on the top surface to avoid rotation in $\theta$ and translations in axial and radial directions. The contraction/expansion of the different designs was simulated by applying a load as a time-variant linear pressure with a peak of $-21 \mathrm{kPa}$. The load was imposed on the channels wall for model $M_{1}, M_{2}$ and $M_{3}$ and on the external surface for model $M_{4}$. To avoid convergence problems, a specific stabilization routine based on the maximum energy dissipation ratio criterion was implemented for each simulation. The principle of the routine is based on a restart process of the simulation in case of non convergence of the solution. The routine imposes a simulation restart at the closest stable substep with an increment energy dissipation ratio. This approach grants to reach simulation convergence with the smallest stabilization ratio possible. The simulations were used to evaluate the following parameters:
- longitudinal displacement $\left(\Delta z_{s i m}\right)$;

- radial displacement $\left(\Delta r_{\text {sim }}\right)$;

- twist angle $\left(\Delta \theta_{\text {sim }}\right)$;

- ejection fraction $\left(\mathrm{EF}_{\text {sim }}\right)$.

The first three parameters were computed by analyzing the displacement of five selected nodes: a node was positioned on the external surface $\left(n_{0}\right)$ to evaluate $\Delta z_{\text {sim }}$ (Figure 3a) while four nodes were positioned on the internal surface $\left(n_{1}\right.$, $n_{2}, n_{3}$ and $\left.n_{4}\right)$ to evaluate $\Delta r_{\text {sim }}$ and $\Delta \theta_{\text {sim }}$ (Figure $3 \mathrm{~b}$ ).
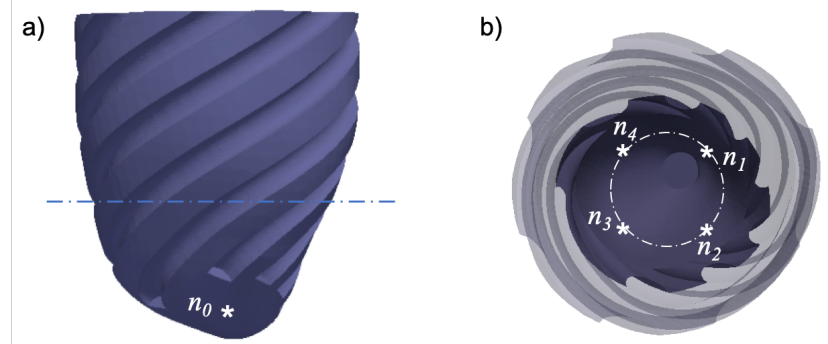

Figure 3. Example of nodes position reported on $D_{4}$.

The $\Delta z_{\text {sim }}$ corresponds to the longitudinal variation of the $n_{0}$ node. The $\Delta r_{\text {sim }}$ is the average value of the radial displacement of $n_{1}, n_{2}, n_{3}$ and $n_{4}$.

Concerning the $\Delta \theta_{\text {sim }}$ measure, the following Equation 2 was used:

$$
\begin{array}{rl}
\Delta \theta_{\text {sim }}=0 & 0.5\left(\arccos \left(\left.\left.\overline{n_{1} n_{3}}\right|_{s y s} \cdot \overline{n_{1} n_{3}}\right|_{d i a}\right)+\right. \\
& \left.\arccos \left(\left.\left.\overline{n_{2} n_{4}}\right|_{s y s} \cdot \overline{n_{2} n_{4}}\right|_{\text {dia }}\right)\right)
\end{array}
$$

Regarding the $\mathrm{EF}_{\text {sim }}$, the corresponding equation used for the segmentation phase was adopted (Equation 1). The results of simulations were compared with the corresponding patient specific values and with data from literature.

\section{Experimental testing}

After assessing the designed prototype model on the basis of the performances from the FEM simulations, the final CAD geometry was manufactured in TangoBlackPlus via $3 \mathrm{D}$ printing Polyjet technology. To assess the $3 \mathrm{D}$ printed geometry behaviour with the MRI environment (compatibility and presence of imaging artifacts), MRI images of the realized device were acquired with the same protocol used for the volunteer imaging. The device was submerged in water during the examination to enhance the MR signal quality. Due to the two different loading strategies required in the design phase, a specific experimental setup, able to work in two configurations was designed. The first configuration required to apply a negative pressure on the channels of the designs $D_{1}, D_{2}$ and $D_{3}$, while the second one required a negative pressure acting on the epicardium surface of design $D_{4}$. The pressure wave load was controlled by a feed-backed 3-way electrovalve (VPPM electrovalve, Festo), permitting to manage the vacuum conditions. The electrovalve directly interconnected the chamber to a vacuum line and modulated the pressure. Figure 4 depicts the overall structure of the experimental setup. 


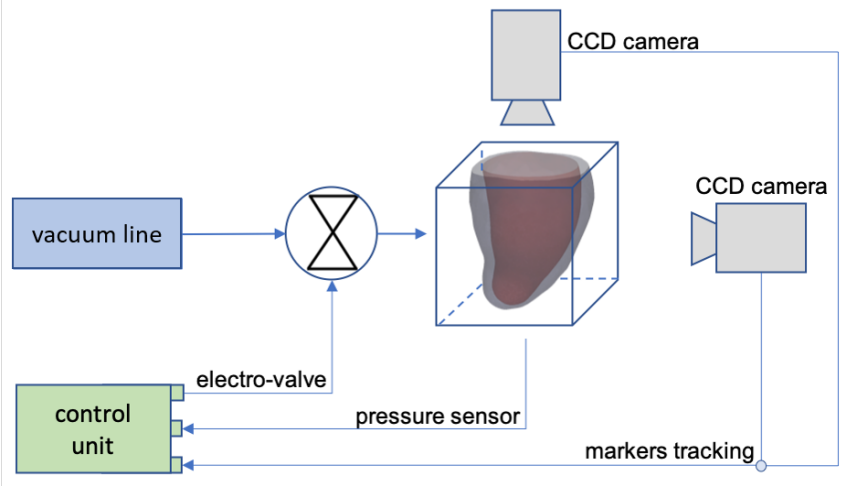

Figure 4. Scheme of the experimental setup.

A catheter pressure transducer (TruWave, Edwards LifeSciences) was used to monitor the applied load pressure. In order to compare the experimental results with those from the numerical simulations, five optical markers $\left(m_{0}, m_{1}, m_{2}\right.$, $m_{3}$ and $m_{4}$ ) were positioned according to the locations of the relative nodes $\left(n_{0}, n_{1}, n_{2}, n_{3}\right.$ and $\left.n_{4}\right)$. The resulting marker coordinates were recorded (30 fps and resolution of $0.03 \mathrm{~mm} / \mathrm{px}$ ) through two digital cameras. The $\Delta z_{\text {exp }}$, $\Delta r_{e x p}$ and $\Delta \theta_{\text {exp }}$ were evaluated as previously described in the numerical section. In this case, the EF was evaluated according to Equation 1. The endocardium volumes were measured by directly evaluating the water mass contained in the ventricle at null pressure and at peak load. The sensor acquisition, the marker tracking and the pressure control were managed by Real-Time control unit and a host PC with a custom application developed in LabView.

\section{Results and Discussions}

\section{Material testing}

The uniaxial tensile tests revealed a linear elastic behaviour with a Young modulus equal to $0.5 \pm 0.017 \mathrm{MPa}$ and a ultimate elongation value equal to $44 \pm 1.1 \%$.

\section{Finite element simulation}

The FEM models were successfully implemented for all the cases. In Figure 5 the resultant displacement fields on the relative deformed shape for the $M_{4}$ model is displayed.

Table 1. Design performance comparison according to FEM simulations with errors relative to physiological values. For references (Ref): $\S$ (Rüssel et al. (2009)), $\dagger$ (Bravo et al. (2009))

\begin{tabular}{lcccccc}
\hline Variable & $D_{1}$ & $D_{2}$ & $D_{3}$ & $D_{4}$ & $S e g$ & $R e f$ \\
\hline$\Delta \theta\left(^{\circ}\right)$ & 7.5 & 1.9 & 2.7 & 9.2 & - & $8.1 \pm 1.1^{\S}$ \\
$E F(\%)$ & 53.5 & 39.7 & 58.0 & 63.9 & 68.8 & $53-77^{\dagger}$ \\
\hline
\end{tabular}

The relative $\Delta \theta$ and $\mathrm{EF}$ values for the $M_{1-4}$ models are reported in Table 1. From these, it is possible to compare the different performances of the models with the physiological data from segmentation and from the literature (Rüssel et al. (2009); Bravo et al. (2009)). The design $D_{2}$ and $D_{3}$ exhibited low twist angles, not comparable with the ones from literature. On the contrary, $D_{1}$ and $D_{4}$ showed values closer to the physiological target. The adoption of layers of channels inside the thickness between epicardium and endocardium appeared to be ineffective in terms of twist rotation. On the other hand, the reproduction of structures (channels for $D_{1}$ and grooves for $D_{4}$ ) directly on the epicardium produced higher values of $\Delta \theta$.

Concerning the EF, the designs relying on channels (from $D_{1}$ to $D_{3}$ ) reached lower values in comparison with the one relying on external grooves $\left(D_{4}\right)$. The higher performance was reached by $D_{4}$, with a $63.9 \%$ value. This percentage was the closer one to the EF from patient's segmented data. It is also within the physiological range reported by Bravo and colleagues (Bravo et al. (2009)). Given these evidences, $D_{4}$ design exhibited the highest performances both in terms of $\Delta \theta$ and EF. Starting from these results, the $D_{4}$ design was considered as the effective configuration to adopt for the pump realization. The usage of external grooves instead of channels implies wider loading surfaces and, consequently, higher volumetric variations.

\section{Experimental testing}

The designed pump prototype was successfully fabricated. The grooves feature was completely reproduced on the epicardium surface as planned. In order to facilitate assembly of the model inside the experimental setup, a rigid support part was realized in VeroClearWhite on the top surface. Figure 6 shows the resulting MR images of the fabricated design. The image quality is satisfactory and permits the correct monitoring of the device without artifacts.

The experimental setup was configured to work in negative pressure regime as described in the previous section. The pressurization chamber was realized in polymethylmethacrylate. This fabrication choice presented two main advantages: transparency and MRI compatibility (Figure 7). On the basis of the experimental positions of the markers, the relative displacements and the twist angle were successfully recorded both on the radial and longitudinal plane and they were compared with the relative values from the FEM simulation (Figure 8). It is possible to evaluate that the experimental trend is in accordance with the data from the FEM simulation with an average error of $13 \%, 14 \%$ and $16 \%$ for $\Delta r, \Delta z$ and $\Delta \theta$ respectively. The experimental EF $(64.4 \%)$ resulted to be comparable with the value from the simulation $(63.9 \%)$. The experimental values appeared to be higher in comparison with the simulated ones for all the variables considered. This behavior can be explained by the usage of artificial nodal dampers introduced in the FEM simulation and required to limit the structural buckling effect encountered during the pump loading: this approach improved the stability but caused a reduction of the final displacement. However, the overall experimental trend was reproduced. Additionally, the experimental EF and the maximum $\Delta \theta$ values $\left(64.4 \%\right.$ and $11.7^{\circ}$, respectively) are comparable with both physiological data from segmentation $(68.8 \%)$ and from literature $\left(53-77 \%\right.$ and $8.1 \pm 1.1^{\circ}$, respectively). The relative error is equal to $2 \%$ for the $\mathrm{EF}$ and equal to $17 \%$ for the $\Delta \theta$.

Limitations and future work - In general, the experimental evidence revealed a good accordance with the results from simulations, confirming the performances of the preliminary design. The setup demonstrated the possibility to have satisfactory values of $\mathrm{EF}$ and $\Delta \theta$ by using a pneumatic 
a)

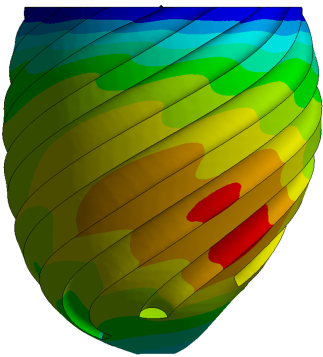

d)

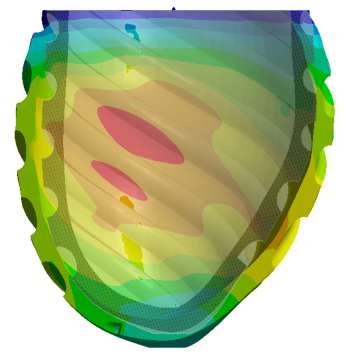

b)

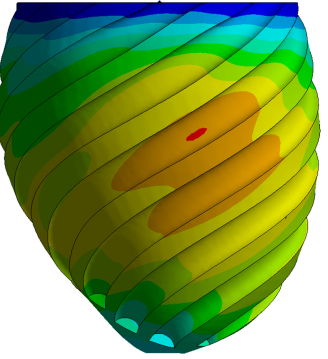

e)

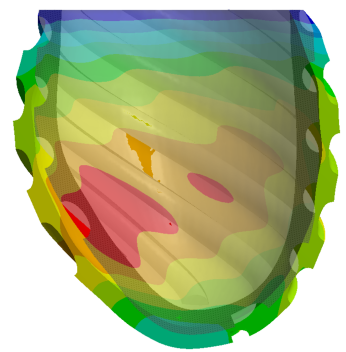

c) $\pi$

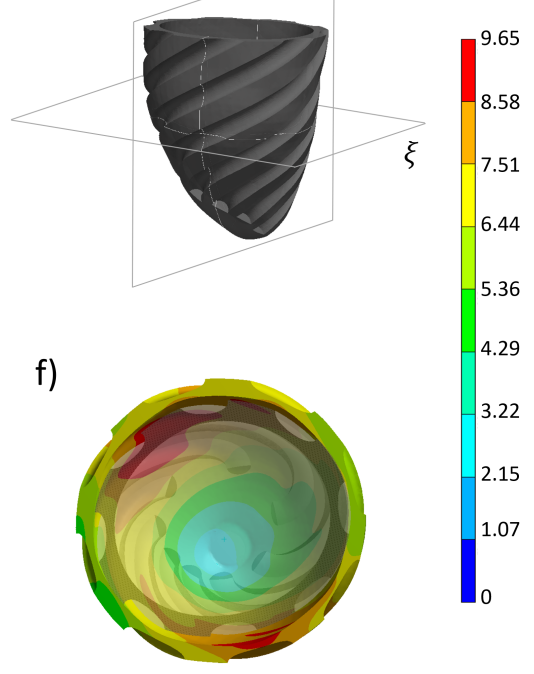

Figure 5. Total displacement maps for $M_{4}$ : left (a) and right (b) side views. To permit the comparison with the undeformed shape, the $\pi$ and $\xi$ planes (c) were used to obtain side (d-e) and top (f) sections; units are in $\mathrm{mm}$.
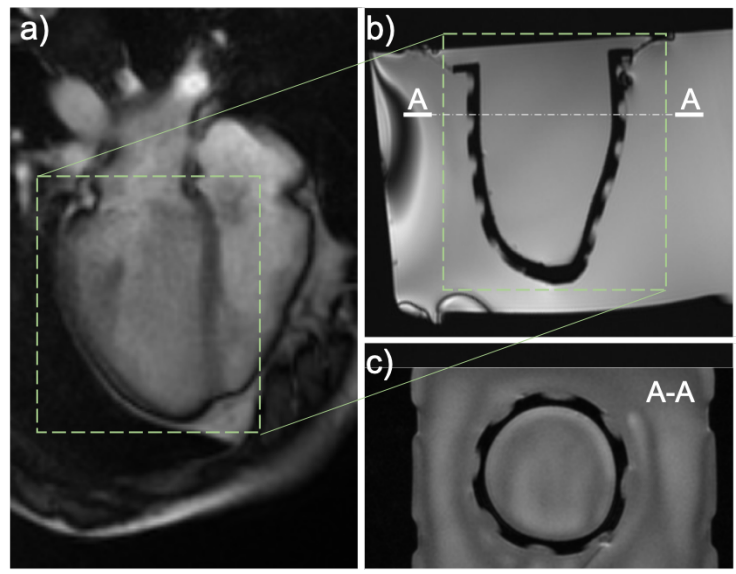

Figure 6. Example of MR images: long axis section of patient LV (a); long (b) and short (c) axis of the 3D printed model.

\section{a)}

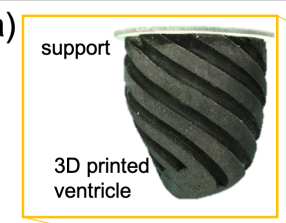

b)

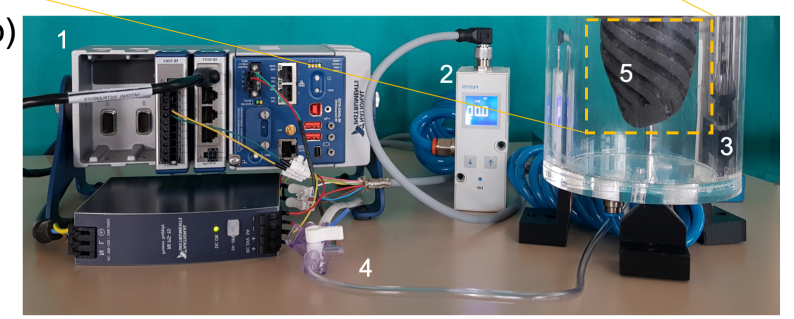

Figure 7. 3D printed model of $D_{4}$ (a) and experimental setup in configuration for $D_{4}$ (b) with indication for the control unit (1), electrovalve (2), pressurization chamber (3), pressure sensor (4) and 3D printed ventricle (5).

actuation approach. Limitations within this study are present though. The choice of the material for manufacturing was limited to polyurethanes. The usage of this material has the intrinsic issue of not being transparent to light and consequently not usable for experiments where an optical inspection is required. Another limitation is the absence of a complete fluid dynamic characterization of the pump system. The system currently does not include valves to fully replicate a functional mock circulatory loop. However, the pneumatic actuation of the chamber and the effectiveness of the helical grooves design was successfully demonstrated with remarkable results. This achievement opens future developments in our study that will be focused in moving from a design prototype to a more complete pumping system.

\section{Conclusion}

The proposed approach demonstrates the feasibility to fabricate a ventricle pump able to reproduce the ejection fraction and twist rotation values comparable with the physiological ones. These results were obtained by means of a specific design, characterized by a set of helical grooves on the epicardium surface. A combined approach of in-silico and in-vitro analyses was implemented. A first prototype was then manufactured and successfully actuated, revealing that the in-vitro performances are in accordance with the insilico trend. Our system is fully MRI compatible thanks to the realization through $3 \mathrm{D}$ printing technique of polymeric material and the actuation via vacuum. The adoption of a $3 \mathrm{D}$ printing technology offers the possibility to make LV models of different sizes, coping with a wide range of patient population from pediatric to adults. Additional advantages are: the reduction of setting up times, the increase of the reproducibility as well as the simplification of the setup management. These results are a valid benchmark for the development of a complete pumping system. The application of this type of actuator showed to be a promising approach in the field of cardiovascular device validation, with the potential to reproduce flow helicity. This aspect play a key role for the physiological system both at valve and aortic level in terms of energy balance and 
a)

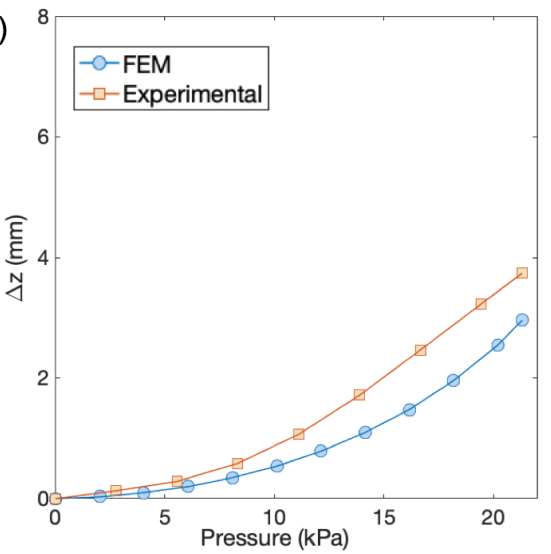

b)

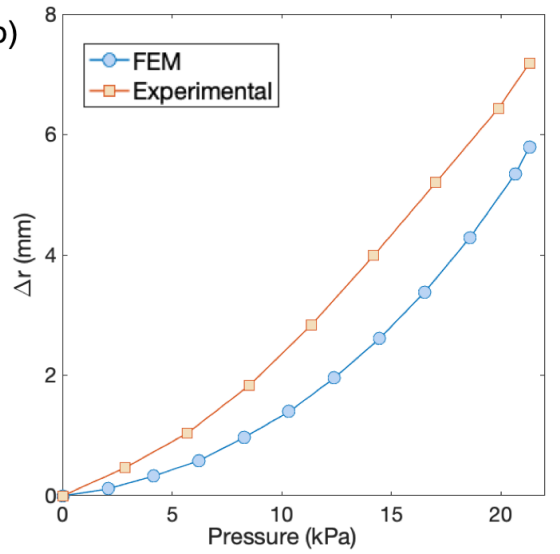

c)

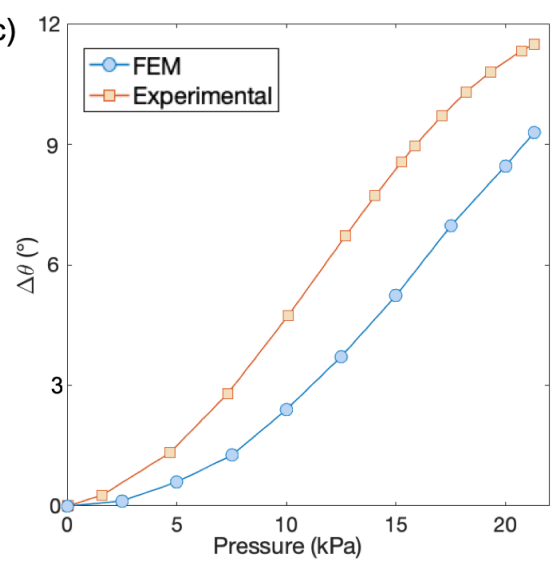

Figure 8. Comparison between FEM and experimental results for $\Delta z(\mathrm{a}), \Delta r$ (b) and $\Delta \theta$ (c) variables as a function of the absolute value of the load.

dissipation. The possibility to integrate our setup with a MRI scan brings added value for further in-vitro studies, including the monitoring of the physiological complexity of haemodynamic flow at bench-test level.

\section{References}

Aquaro GD, Camastra G, Monti L, Lombardi M, Pepe A, Castelletti S, Maestrini V, Todiere G, Masci P, Di Giovine G, Barison A, Dellegrottaglie S, Perazzolo Marra M, Pontone G, Di Bella G and behalf of the working group Applicazioni della Risonanza Magnetica of the Italian Society of Cardiology O (2017) Reference values of cardiac volumes, dimensions, and new functional parameters by mr: A multicenter, multivendor study. Journal of Magnetic Resonance Imaging 45(4): 1055-1067.
Beladan CC, Călin A, Roşca M, Ginghină C and Popescu BA (2014) Left ventricular twist dynamics: principles and applications. Heart 100(9): 731-740.

Bravo P, Chien D, Javadi M, Merrill J and Bengel F (2009) Normal ranges for left ventricular ejection fraction and volumes from electrocardiographically gated 82rb cardiac pet/ct using four different softwares. Journal of Nuclear Medicine 50(supplement 2): 480.

Celi S, Martini N, Pastormerlo LE, Positano V and Berti S (2017) Multimodality imaging for interventional cardiology. Current Pharmaceutical Design 23: 1-16. In press.

Chaudhury RA, Atlasman V, Pathangey G, Pracht N, Adrian RJ and Frakes DH (2016) A high performance pulsatile pump for aortic flow experiments in 3-dimensional models. Cardiovascular Engineering and Technology 7(2): 148-158.

Gasparotti E, Vignali E, Losi P, Scatto M, Marco Fanni B, Soldani G, Landini L, Positano V and Celi S (2018) A $3 \mathrm{~d}$ printed melt-compounded antibiotic loaded thermoplastic polyurethane heart valve ring design: an integrated framework of experimental material tests and numerical simulations. International Journal of Polymeric Materials and Polymeric Biomaterials : 1-10.

Jaworek M, Piola M, Lucherini F, Gelpi G, Castagna M, Lentini G, Antona C, B Fiore G and Vismara R (2017) Functional tricuspid regurgitation model in a beating heart platform. ASAIO Journal 63: 1.

Lanzarone E, Vismara R and Fiore GB (2009) A new pulsatile volumetric device with biomorphic valves for the in vitro study of the cardiovascular system. Artificial Organs 33(12): 10481062.

Leopaldi A, Vismara R, van Tuijl S, Redaelli A, van de Vosse F, Fiore G and Rutten M (2015) A novel passive left heart platform for device testing and research. Medical Engineering Physics 37(4): 361 - 366.

Marconi S, Lanzarone E, Bogerijen G, Conti M, Secchi F, Trimarchi $\mathrm{S}$ and Auricchio F (2018) A compliant aortic model for in vitro simulations: Design and manufacturing process. Medical Engineering Physics 59.

Marone E, Auricchio F, Marconi S, Conti M, Rinaldi L, Pietrabissa A and Argenteri A (2018) Effectiveness of 3d printed models in the treatment of complex aortic diseases. The Journal of cardiovascular surgery 59.

Mechoor RR, Schmidt T and Kung E (2016) A real-time programmable pulsatile flow pump for in vitro cardiovascular experimentation. Journal of biomechanical engineering 138 11.

Morbiducci U, Ponzini R, Rizzo G, Cadioli M, Esposito A, Montevecchi FM and Redaelli A (2011) Mechanistic insight into the physiological relevance of helical blood flow in the human aorta: an in vivo study. Biomechanics and Modeling in Mechanobiology 10(3): 339-355.

Nakatani S (2011) Left ventricular rotation and twist: Why should we learn? In: Journal of cardiovascular ultrasound.

Roche ET, Horvath MA, Wamala I, Alazmani A, Song SE, Whyte W, Machaidze Z, Payne CJ, Weaver JC, Fishbein G, Kuebler J, Vasilyev NV, Mooney DJ, Pigula FA and Walsh CJ (2017) Soft robotic sleeve supports heart function. Science Translational Medicine 9(373).

Rüssel IK, Gtte MJ, Bronzwaer JG, Knaapen P, Paulus WJ and van Rossum AC (2009) Left ventricular torsion: An expanding 
role in the analysis of myocardial dysfunction. JACC: Cardiovascular Imaging 2(5): 648 - 655.

Sengupta PP, Khandheria BK and Narula J (2008) Twist and untwist mechanics of the left ventricle. Heart Failure Clinics 4(3): 315 - 324. Function Follows Form.

Taylor C, Dziczkowski Z and E Miller G (2012) Automation of the harvard apparatus pulsatile blood pump. Journal of Medical Devices 6: 045002.

Tsai W and Savaş Ö (2010) Flow pumping system for physiological waveforms. Medical \& Biological Engineering \& Computing 48(2): 197-201. 